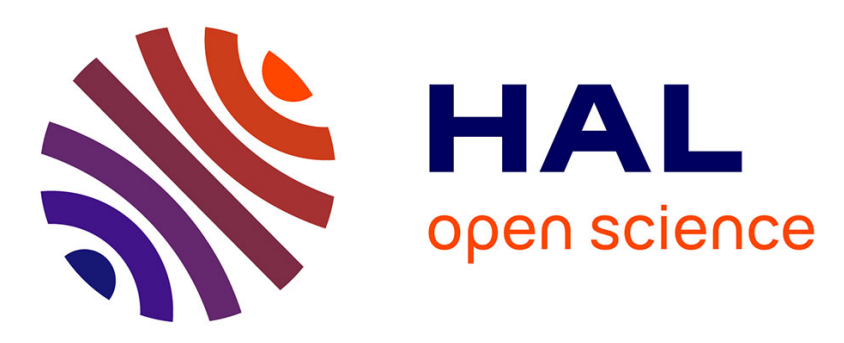

\title{
Fresh Air Fraction Control in Engines Using Dynamic Boundary Stabilization of LPV Hyperbolic Systems
}

Felipe Castillo, Emmanuel Witrant, Christophe Prieur, Vincent Talon, Luc Dugard

\section{- To cite this version:}

Felipe Castillo, Emmanuel Witrant, Christophe Prieur, Vincent Talon, Luc Dugard. Fresh Air Fraction Control in Engines Using Dynamic Boundary Stabilization of LPV Hyperbolic Systems. IEEE Transactions on Control Systems Technology, 2015, 23 (3), pp.963-974. 10.1109/TCST.2014.2356858 . hal-01144114

\author{
HAL Id: hal-01144114 \\ https://hal.science/hal-01144114
}

Submitted on 21 Apr 2015

HAL is a multi-disciplinary open access archive for the deposit and dissemination of scientific research documents, whether they are published or not. The documents may come from teaching and research institutions in France or abroad, or from public or private research centers.
L'archive ouverte pluridisciplinaire HAL, est destinée au dépôt et à la diffusion de documents scientifiques de niveau recherche, publiés ou non, émanant des établissements d'enseignement et de recherche français ou étrangers, des laboratoires publics ou privés. 


\title{
Fresh Air Fraction Control in Engines Using Dynamic Boundary Stabilization of LPV Hyperbolic Systems
}

\author{
Felipe Castillo, Emmanuel Witrant, Christophe Prieur, Vincent Talon and Luc \\ Dugard
}

\begin{abstract}
In this paper, we consider the boundary control of the fresh air mass fraction in a Diesel engine operated with low-pressure exhaust gas recirculation. The air mass fraction transport phenomenon is modeled using a cascade of first-order linear parameter varying (LPV) hyperbolic systems with dynamics associated with their boundary conditions. By means of Lyapunov based techniques, sufficient conditions are derived to guarantee the exponential stability of this class of infinite dimensional systems. We develop a polytopic approach to synthesize a robust boundary control that guarantees the exponential stability for a given convex parameter set. Simulation results illustrate the effectiveness of the proposed boundary control to regulate the mass fraction of fresh air in a Diesel engine.
\end{abstract}

\section{INTRODUCTION}

Regulations of Diesel engine emissions have become stricter, and satisfying simultaneously the emissions legislations and the desired engine drivability objectives is a particularly challenging issue. Although significant improvements were made over the past years, there are still many technical issues that need to be addressed in order to meet the future regulation laws on emissions. The introduction of sophisticated alternative combustion modes such as homogeneous

Felipe Castillo, Emmanuel Witrant, Christophe Prieur and Luc Dugard are with UJF-Grenoble 1/CNRS, Grenoble Image Parole Signal Automatique (GIPSA-lab), UMR 5216, B.P. 46, F-38402 St Martin d'Hères, France. (felipe.castillo-buenaventura, emmanuel.witrant, christophe.prieur, luc.dugard)@gipsa-lab.fr. This work is partially supported by HYCON2 Network of Excellence (Highly-Complex and Networked Control Systems), grant agreement 257462. Felipe Castillo and Vincent Talon are with Renault SAS, 1 Allee Cornuel, 91510 Lardy, France (e-mail: vincent.talon@renault.com) 
charge compression ignition (HCCI), low temperature combustion (LTC) and premixed controlled compression ignition (PCCI) offers a great potential to reduce the engine emissions levels [1] [2] [30]. However, these new modes require specific fueling strategies and in-cylinder conditions, thus creating the need for more complex, reliable and precise control systems and technologies.

Dual-loop exhaust gas recirculation (EGR) with both high (HP) and low-pressure (LP) recirculations is one of the new strategies that can provide the appropriate conditions for multiple combustion modes [22]. Ensuring the adequate in-cylinder conditions is still a difficult task, due to the lack of measurements for EGR flow rates and mass fraction. Several air mass fraction/EGR rate control and estimation methods have been proposed in the literature [14] [20] [34] [37]. However, most of the actual air fraction control techniques are based on zero-dimensional (0D) engine modeling, which does not allow taking into account the air transport inside the engine admission air-path. Indeed, the mass transport causes a degradation of the overall engine emission performance during strong transient conditions. This is mostly due to the LP-EGR, as the distance that the gas travels in the engine air-path is much longer than the one associated with HP-EGR. The control of air fraction taking into account the mass transport time is a significantly less explored subject, although in [6] a non-physical representation of the delay by means of PDES was used to design a predictor-based controller.

The flow transport strongly depends on the engine operating conditions. It has been shown in [13] and [35] that this dependence can be modeled by using a linear parameter varying (LPV) approach. On the other hand, the transport of mass is often modeled by first order hyperbolic partial differential equations (PDEs), as reported in [4], [10] and [31]. An LPV hyperbolic model can thus capture the dynamics of the air mass fraction and be used to solve the associated control problem.

Several results are available in the literature for the control of first order hyperbolic systems. For instance, sufficient conditions for controllability and observability of quasilinear hyperbolic systems have been obtained in [24]. The boundary control of hyperbolic systems has been considered in [17], [16], [26] and [27], among other references. Most results consider that the boundary control can react fast enough in comparison with the waves travel time. More 
precisely, no time response limitation is taken into account at the boundary conditions. For many applications (e.g. [5] [18]), the wave travel can be considered as being much slower than the actuator time response. A static relationship can then be established between the control input and the boundary condition. Nevertheless, there are applications where the dynamics associated with the boundary control cannot be neglected (e.g. the use of LP-EGR to control the fresh air fraction in an engine intake manifold). To address this problem, a discretization of the infinitedimensional system has been used in [7] to apply finite-dimensional control tools. In [10] some sufficient conditions for the exponential stability of hyperbolic systems with linear time invariant (LTI) dynamic boundary conditions have been given. In [28] and [21], a strict time-varying Lyapunov function that allows establishing the asymptotic stability of time-varying hyperbolic systems is formulated for time-invariant boundary conditions. In [32], a multi-model approach (similar to an LPV) with a bilinear matrix inequality has been proposed for the stability of a hyperbolic system representing the flow in an open channel. In [23], backstepping designs for the boundary control of hyperbolic and time delayed systems have been proposed. However, to the best of our knowledge, no boundary control design for LPV hyperbolic systems with LPV boundary conditions has been addressed in the literature.

This article, which is an extension of our previous work [8], focuses on the stabilization of LPV hyperbolic systems with boundary conditions defined by LPV dynamics and its application to the control of the air fraction in the intake manifold of Diesel engines. To demonstrate the asymptotic stability for this class of hyperbolic systems, we use an extension of the strict Lyapunov function formulation presented in [17]. A polytopic approach is developed to synthesize a robust boundary control that guarantees the exponential stability for a given convex parameter set. We obtain sufficient conditions in terms of the boundary conditions to prove Lyapunov stability. This feedback design strategy is used for the boundary control of the fresh air mass fraction in an engine air-path with LP-EGR. The effectiveness of our approach is evaluated in simulation using a 1D model of the engine admission air-path.

This paper is organized as follows: the problem formulation and the main stability results for LPV hyperbolic systems with LPV dynamic behavior at the boundary conditions are presented in Section II. In Section III, an LPV hyperbolic system is used to model the air fraction in the 
engine air-path and an air fraction boundary control is designed using the results of Section II. In Section IV, simulation results are presented to illustrate the effectiveness of the proposed control strategy.

Notation. By the expressions $H \succeq 0, H \preceq 0, H \succ 0$ and $H \prec 0$ we mean that the matrix $H$ is positive semi-definite, negative semi-definite, positive definite and negative definite, respectively. The usual Euclidian norm in $\mathbb{R}^{n}$ is denoted by $|$.$| and the associated matrix norm is denoted$ $\|$.$\| . Given g:[0,1] \rightarrow \mathbb{R}^{n}$, we define its $L^{2}$-norm (when is finite) as:

$$
\|g\|_{L^{2}}=\sqrt{\int_{0}^{1}|g(x)|^{2} d x}
$$

\section{Stability of Parameter-VArying Linear Hyperbolic Systems With Dynamic \\ BOUNDARY CONDITIONS}

Let $n$ be a positive integer, $\Theta$ an open non-empty convex set of $\mathbb{R}^{n}$ and $Z_{\varphi}$ a non empty convex set of $\mathbb{R}^{l}$. Consider the general class of first order LPV hyperbolic systems of order $n$ defined as follows:

$$
\partial_{t} \xi(x, t)+\Lambda(\varphi) \partial_{x} \xi(x, t)=0 \quad \forall x \in[0,1], t \geq 0
$$

where $\xi:[0,1] \times[0,+\infty) \rightarrow \Theta, \varphi$ is a varying parameter vector that takes values in the

parameter space $Z_{\varphi}, \Lambda(\varphi): Z_{\varphi} \rightarrow \mathbb{R}^{n \times n}$ is a diagonal and invertible matrix function (called the characteristic matrix) such that $\Lambda(\varphi)=\operatorname{diag}\left(\lambda_{1}(\varphi), \lambda_{2}(\varphi), \ldots, \lambda_{n}(\varphi)\right), \partial_{t}$ and $\partial_{x}$ denote the partial derivatives with respect to time and space, respectively.

Assumption 1: Assume that the following inequalities hold for all $\varphi \in Z_{\varphi}$ :

$$
0<\lambda_{1}(\varphi)<\ldots<\lambda_{n}(\varphi)
$$

Consider the following dynamic boundary conditions for (1):

$$
\begin{aligned}
& \dot{X}_{c}=A_{c}(\varphi) X_{c}+B_{c}(\varphi) u \\
& \xi(0, t)=C_{c} X_{c}+D_{c} u
\end{aligned}
$$


with

$$
u=K \xi(1, t)
$$

where $X_{c} \in \mathbb{R}^{n_{x}}, A_{c}: Z_{\varphi} \rightarrow \mathbb{R}^{n_{x} \times n_{x}}, B_{c}: Z_{\varphi} \rightarrow \mathbb{R}^{n_{x} \times n}, C_{c} \in \mathbb{R}^{n \times n_{x}}, D_{c} \in \mathbb{R}^{n \times n}, K \in \mathbb{R}^{n \times n}$, $u \in \mathbb{R}^{n}$ and $n_{x} \geq 1$. Define the initial condition for (1) and (3) as:

$$
\left\{\begin{array}{c}
\xi(x, 0)=\xi^{0}(x), \quad \forall x \in[0,1] \\
X_{c}(0)=X_{c}^{0}
\end{array}\right.
$$

where $\xi^{0}(x) \in L^{2}\left((0,1) ; \mathbb{R}^{n}\right)$ and $X_{c}^{0} \in \mathbb{R}^{n_{x}}$. Such an initial condition is required for the existence of a unique classical solution of the Cauchy problem (1) - (5) [19]. In this section, we consider the problem of finding a control gain $K$ and a positive scalar $\mu$ such that the system (1) with boundary conditions (3) and initial condition (5) is exponentially stable for all $\varphi \in Z_{\varphi}$ satisfying the following inequality.

$$
\left\|X_{c}(t)\right\|^{2}+\|\xi(t)\|_{L^{2}(0,1)} \leq b\left(\left\|X_{c}^{0}\right\|^{2}+\left\|\xi^{0}\right\|_{L^{2}(0,1)}\right) e^{-\mu \varrho t}
$$

where $b$ and $\varrho$ are positive scalars.

Let the polytope $Z_{\varphi}$ be defined as follows:

$$
Z_{\varphi}:=\left\{\left[\varphi_{1}, \ldots, \varphi_{l}\right]^{T} \in \mathbb{R}^{l} \mid \varphi_{i} \in\left[\underline{\varphi}_{i}, \bar{\varphi}_{i}\right], \forall i=1, \ldots, l\right\}
$$

for given $l \in \mathbb{N}^{+}$and the parameter extremities $\underline{\varphi}_{i}, \bar{\varphi}_{i}$ (minimum and maximum, respectively). We thus consider that all the admissible values of the vector $\varphi$ are constrained in a hyperrectangle in the parameter space $Z_{\varphi}$. Consider the polytopic linear representation of the parameter varying characteristic matrix for all $\varphi \in Z_{\varphi}[3]$ :

$$
\Lambda(\varphi)=\sum_{i=1}^{N_{\varphi}} \alpha_{i}(\varphi) \Lambda\left(w_{i}\right)
$$

where $w_{i} \in Z_{\varphi}$ are the $N_{\varphi}=2^{l}$ vertices of the polytope formed by all extremities $\left(\bar{\varphi}_{i}\right.$ and $\left.\underline{\varphi}_{i}\right)$ of each varying parameter $\varphi \in Z_{\varphi}, \sum_{i=1}^{2^{l}} \alpha_{i}(\varphi) \Lambda\left(w_{i}\right): Z_{\varphi} \rightarrow \mathbb{R}^{n \times n}$ and $\alpha_{i}(\varphi)$ is a scheduling function $\alpha_{i}: Z_{\varphi} \rightarrow[0,1]$ defined as: 


$$
\alpha_{i}(\varphi)=\frac{\prod_{k=1}^{l}\left|\varphi_{k}-C\left(w_{i}\right)_{k}\right|}{\prod_{k=1}^{l}\left|\bar{\varphi}_{k}-\underline{\varphi}_{k}\right|}
$$

where $C\left(w_{i}\right)_{k}$ is the $k t h$ component of the vector $C\left(w_{i}\right)$ defined as:

$$
C\left(w_{i}\right)_{k}=\left\{\begin{array}{c}
\bar{\varphi}_{k}, \text { if }\left(w_{i}\right)_{k}=\underline{\varphi}_{k} \\
\underline{\varphi}_{k}, \text { otherwise }
\end{array}\right.
$$

The scheduling functions $\alpha_{i}$ have the following properties [3]:

$$
\alpha_{i}(\varphi) \geq 0, \quad \sum_{i=1}^{N_{\varphi}} \alpha_{i}(\varphi)=1
$$

The polytopic representation (8) can also be considered for the matrices $A_{c}(\varphi)$ and $B_{c}(\varphi)$ of the boundary conditions (3) using the same scheduling function (9). Define the polytopic linear representation (PLR) of the parameter varying hyperbolic system (1) with boundary conditions (3) as follows:

$$
\begin{aligned}
& \partial_{t} \xi(x, t)+\sum_{i=1}^{N_{\varphi}} \alpha_{i}(\varphi) \Lambda\left(w_{i}\right) \partial_{x} \xi(x, t)=0 \\
& \forall \varphi \in Z_{\varphi}, \quad \forall x \in[0,1], \quad t \geq 0
\end{aligned}
$$

with boundary conditions

$$
\begin{aligned}
& \dot{X}_{c}=\sum_{i=1}^{N_{\varphi}} \alpha_{i}(\varphi) A_{c}\left(w_{i}\right) X_{c}+\sum_{i=1}^{N_{\varphi}} \alpha_{i}(\varphi) B_{c}\left(w_{i}\right) u \\
& \xi(0, t)=C_{c} X_{c}+D_{c} u
\end{aligned}
$$

Based on the PLR (12) - (13), the following theorem states a sufficient condition to ensure the exponential stability for system (1) with boundary conditions (3) and initial condition (5) for all $\varphi \in Z_{\varphi}$.

Theorem 1. [Stability analysis] Along with Assumption 1, assume that there exists two diagonal positive definite matrices $P_{1} \in \mathbb{R}^{n_{x} \times n_{x}}$ and $P_{2} \in \mathbb{R}^{n \times n}$ and a scalar $\mu>0$ such that the following matrix inequality is satisfied, for all $i=1, \ldots, N_{\varphi}$, 


$$
M_{i}=\left[\begin{array}{c|c}
A_{c}\left(w_{i}\right)^{T} P_{1}+P_{1} A_{c}\left(w_{i}\right)+C_{c}^{T} \Lambda\left(w_{i}\right) P_{2} C_{c} & P_{1} B_{c}\left(w_{i}\right) K+C_{c}^{T} \Lambda\left(w_{i}\right) P_{2} D_{c} K \\
+\mu \Lambda\left(w_{i}\right) P_{1} & \\
\hline K^{T} B_{c}\left(w_{i}\right)^{T} P_{1}+K^{T} D_{c}^{T} \Lambda\left(w_{i}\right) P_{2} C_{c} & K^{T} D_{c}^{T} \Lambda\left(w_{i}\right) P_{2} D_{c} K-e^{-\mu} \Lambda\left(w_{i}\right) P_{2}
\end{array}\right] \preceq 0
$$

Then there exist two constant scalars $a>0$ and $b>0$ such that, for all $\xi^{0} \in L^{2}\left((0,1) ; \mathbb{R}^{n}\right)$ and $X_{c}^{0} \in \mathbb{R}^{n_{x}}$, the solution of (1), (3) and (5) satisfies, for all $t \geq 0$

$$
\left\|X_{c}(t)\right\|^{2}+\|\xi(t)\|_{L^{2}(0,1)} \leq b e^{-a t}\left(\left\|X_{c}^{0}\right\|^{2}+\left\|\xi^{0}\right\|_{L^{2}(0,1)}\right)
$$

Proof: Given the diagonal positive definite matrices $P_{1}$ and $P_{2}$, consider (as an extension of the Lyapunov function proposed in [17]) the quadratic strict Lyapunov function candidate defined for all continuously differentiable functions $\xi:[0,1] \rightarrow \Theta$ as:

$$
V\left(\xi, X_{c}\right)=X_{c}^{T} P_{1} X_{c}+\int_{0}^{1}\left(\xi^{T} P_{2} \xi\right) e^{-\mu x} d x
$$

where $\mu$ is a positive scalar. Computing the time derivative $\dot{V}$ of $V$ along the classical $C^{1}$ solutions of (1) with boundary conditions (3) and initial condition (5), yields to the following:

$$
\dot{V}=\dot{X}_{c}^{T} P_{1} X_{c}+X_{c}^{T} P_{1} \dot{X}_{c}+\int_{0}^{1}\left(\dot{\xi}^{T} P_{2} \xi+\xi^{T} P_{2} \dot{\xi}\right) e^{-\mu x} d x
$$

After integration by parts and taking into account the polytopic linear representation (12) and (13), the following is obtained:

$$
\begin{aligned}
\dot{V}= & \sum_{i=1}^{2^{l}} \alpha_{i}(\varphi)\left[\left(X_{c}^{T}\left(A_{c}\left(w_{i}\right)^{T} P_{1}+P_{1} A_{c}\left(w_{i}\right)\right) X_{c}\right)+\left(\xi(1)^{T} K^{T} B_{c}\left(w_{i}\right)^{T} P_{1} X_{c}\right.\right. \\
& \left.\left.\quad+X_{c}^{T} P_{1} B_{c}\left(w_{i}\right) K \xi(1)\right)-\left.\left[e^{-\mu x} \xi^{T} \Lambda\left(w_{i}\right) P_{2} \xi\right]\right|_{0} ^{1}-\mu \int_{0}^{1}\left(\xi^{T} \Lambda\left(w_{i}\right) P_{2} \xi\right) e^{-\mu x} d x\right]
\end{aligned}
$$

where $\xi(1)=\xi(1, t)$. The previous equation can be written using the boundary conditions (3) as follows: 


$$
\begin{aligned}
\dot{V}= & \sum_{i=1}^{2^{l}} \alpha_{i}(\varphi)\left[\left(X_{c}^{T}\left(A_{c}\left(w_{i}\right)^{T} P_{1}+P_{1} A_{c}\left(w_{i}\right)\right) X_{c}\right)+\left(\xi(1)^{T} K^{T} B_{c}\left(w_{i}\right)^{T} P_{1} X_{c}\right.\right. \\
& \left.+X_{c}^{T} P_{1} B_{c}\left(w_{i}\right) K \xi(1)\right)-e^{-\mu} \xi(1)^{T} \Lambda\left(w_{i}\right) P_{2} \xi(1)+X_{c}^{T} C_{c}^{T} \Lambda\left(w_{i}\right) P_{2} C_{c} X_{c} \\
& +X_{c}^{T} C_{c}^{T} \Lambda\left(w_{i}\right) P_{2} D_{c} K \xi(1)+\xi(1)^{T} K^{T} D_{c}^{T} \Lambda\left(w_{i}\right) P_{2} C_{c} X_{c} \\
& \left.+\xi(1)^{T} K^{T} D_{c}^{T} \Lambda\left(w_{i}\right) P_{2} D_{c} K \xi(1)-\mu \int_{0}^{1}\left(\xi^{T} \Lambda\left(w_{i}\right) P_{2} \xi\right) e^{-\mu x} d x\right] \\
& =\sum_{i=1}^{2^{l}} \alpha_{i}(\varphi)\left[\begin{array}{c}
-\mu X_{c}^{T} \Lambda\left(w_{i}\right) P_{1} X_{c}-\mu \int_{0}^{1}\left(\xi^{T} \Lambda\left(w_{i}\right) P_{2} \xi\right) e^{-\mu x} d x \\
\end{array}\right. \\
& {\left[\begin{array}{c}
X_{c} \\
\xi(1)
\end{array}\right]^{T}\left[\begin{array}{c}
X_{c} \\
\xi(1)
\end{array}\right] }
\end{aligned}
$$

where the matrix $M_{i}$ is defined as in (14). The definition $\alpha_{i} \geq 0$ and the matrix inequality $M_{i} \preceq 0$ from (14) imply that the last term of (19) is always negative or zero. This gives the following inequality:

$$
\dot{V} \leq \sum_{i=1}^{2^{l}} \alpha_{i}(\varphi)\left[-\mu X_{c}^{T} \Lambda\left(w_{i}\right) P_{1} X_{c}-\mu \int_{0}^{1}\left(\xi^{T} \Lambda\left(w_{i}\right) P_{2} \xi\right) e^{-\mu x} d x\right]
$$

From (2) it can be proved that there always exists a $\varrho>0$ such that $\Lambda(\varphi)-\varrho I^{n \times n} \succ 0$ (e.g. $\varrho$ could be the smallest eigenvalue of $\Lambda(\varphi)$ over $Z_{\varphi}$ ). Moreover, the diagonality of $P_{1}, P_{2}$ and $\Lambda$ implies that:

$$
\dot{V} \leq-\mu \varrho V\left(\xi, X_{c}\right)
$$

Therefore, the function (16) is a Lyapunov function for the hyperbolic system (1) with boundary conditions (3).

By integrating the inequality (21) from 0 to $t$, we obtain the following:

$$
V(t) \leq V(0) e^{-\mu \varrho t}
$$

To obtain the final result (15), we bound the Lyapunov function as follows: 


$$
\begin{aligned}
& \min \left\{\lambda_{\min }\left(P_{1}\right), \lambda_{\min }\left(P_{2}\right)\right\}\left(\left\|X_{c}(t)\right\|^{2}+\|\xi(t)\|_{L^{2}(0,1)}\right) \leq \\
& V(t) \leq \max \left\{\lambda_{\max }\left(P_{1}\right), \lambda_{\max }\left(P_{2}\right)\right\}\left(\left\|X_{c}(t)\right\|^{2}+\|\xi(t)\|_{L^{2}(0,1)}\right)
\end{aligned}
$$

where $\lambda_{\min }$ and $\lambda_{\max }$ are the minimum and maximum eigenvalues of the considered matrices, respectively. Then, using (22) together with (23) gives:

$$
\left\|X_{c}(t)\right\|^{2}+\|\xi(t)\|_{L^{2}(0,1)} \leq \frac{\max \left\{\lambda_{\max }\left(P_{1}\right), \lambda_{\max }\left(P_{2}\right)\right\}}{\min \left\{\lambda_{\min }\left(P_{1}\right), \lambda_{\min }\left(P_{2}\right)\right\}}\left(\left\|X_{c}^{0}\right\|^{2}+\left\|\xi^{0}\right\|_{L^{2}(0,1)}\right) e^{-\mu \varrho t}
$$

which implies that $a=\mu \varrho$ and $b=\max \left\{\lambda_{\max }\left(P_{1}\right), \lambda_{\max }\left(P_{2}\right)\right\} / \min \left\{\lambda_{\min }\left(P_{1}\right), \lambda_{\min }\left(P_{2}\right)\right\}$ in (15).

Note that the matrix inequality (14) considers, through the Lyapunov matrices $P_{1}$ and $P_{2}$, the dynamic coupling between the system and its boundary conditions. Inequality (14) along with (21) implies that $\mu$ is a tuning parameter of the controller design as it explicitly enables to set the convergence speed of the Lyapunov function. Another interesting convergence feature can be deduced from (21): a faster convergence is obtained for larger values of $\varrho$. This implies that hyperbolic systems with high convective velocities converge faster, which is physically consistent.

The following corollary gives a sufficient condition for the design of a stabilizing controller for the particular case where $C_{c}$ is a diagonal matrix, $D_{c}=0$ and $n=n_{x}$.

Corollary 1. [Design of a stabilizing controller] Along with Assumption 1, if $C_{c}$ is diagonal and $D_{c}=0$ and if there exists a diagonal positive definite matrix $Q \in \mathbb{R}^{n \times n}$ and a scalar $\mu>0$ such that the following linear matrix inequality is satisfied, for all $i \in 1, \ldots, N_{\varphi}$,

$$
\left[\begin{array}{cc}
Q A_{c}\left(w_{i}\right)^{T}+A_{c}\left(w_{i}\right) Q+C_{c} \Lambda\left(w_{i}\right) Q C_{c}+\mu \Lambda\left(w_{i}\right) Q & B_{c}\left(w_{i}\right) Y \\
Y^{T} B_{c}\left(w_{i}\right)^{T} & -e^{-\mu} \Lambda\left(w_{i}\right) Q
\end{array}\right] \preceq 0
$$

where $Y=K Q$, then there exist two constants $\alpha>0$ and $M>0$ such that, for all $\xi^{0} \in$ $L^{2}\left((0,1) ; \mathbb{R}^{n}\right)$ and $X_{c}^{0} \in \mathbb{R}^{n}$, the solution of (1) with boundary conditions (3) and initial condition (5) satisfies (15) for all $t \geq 0$. 
Proof: The proof of Corollary 1 can be found in [8].

This corollary is interesting because, for systems where $C_{C}$ is diagonal and $D_{c}=0$, it provides a constructive approach to obtain the boundary control gain $K$ using convex optimization algorithms after determining a suitable value of $\mu>0$ (e.g. chosen to obtain a good performance versus robustness trade-off for the system considered).

\section{Fresh Air Mass Fraction CONTROL}

For the air fraction boundary control design problem, consider the admission air-path of a Diesel engine with low pressure exhaust gas recirculation (LP-EGR) presented in Figure 1.

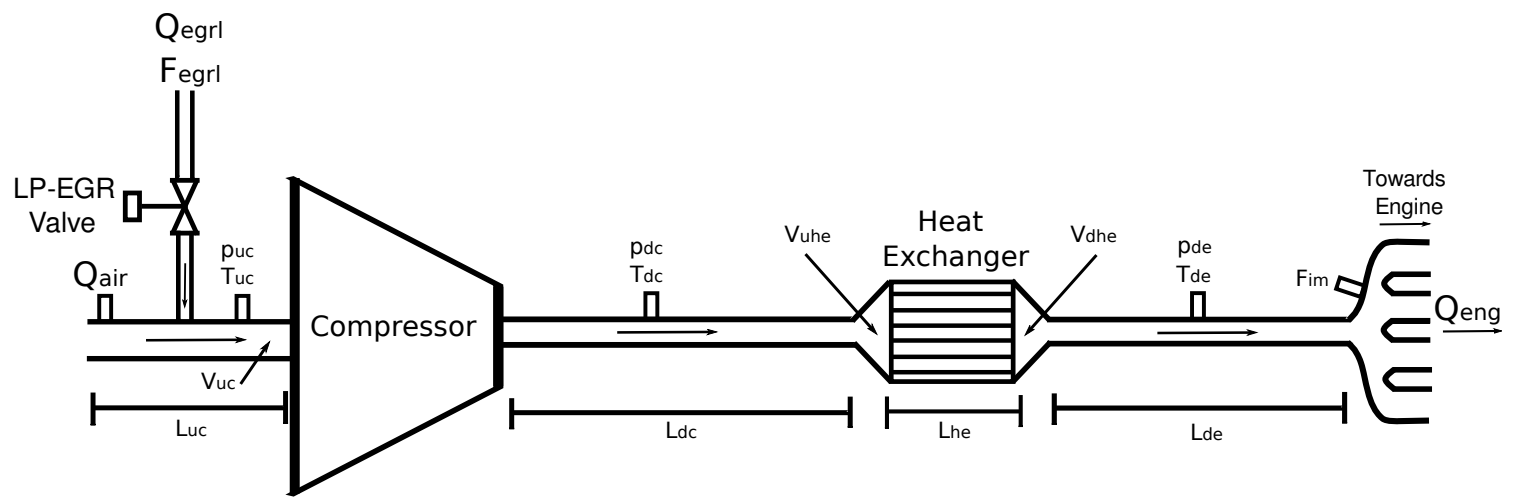

Fig. 1: Schematic of a Diesel engine admission air-path

A fresh air mass flow rate $Q_{a i r}$ enters the admission air-path where it is mixed upstream (subscript $u$ ) the compressor (subscript $c$ ) with the LP-EGR (characterized by its mass flow rate $Q_{\text {egrl }}$ and air fraction $\left.F_{\text {egrl }}\right)$. The exhaust gas recirculation mass flow rate is controlled by the position of the LP-EGR valve. $F_{\text {egrl }}$ depends on the engine operating conditions and is considered here as a known exogenous input since this quantity is measured in production engines. The compressor increases the enthalpy of the gas which results in an increase of the compressor downstream (subscript $d$ ) gas pressure and temperature $p_{d c}$ and $T_{d c}$, respectively (see Figure 1). To increase the gas mass in the engine cylinders and therefore the engine power, the gas coming from the compressor is cooled down by a heat exchanger, which increases the gas density and therefore 
the mass inside the cylinders. The pressure and temperature downstream of the heat exchanger are denoted as $p_{d h e}$ and $T_{d h e}$, respectively. Finally, the gas travels from the heat exchanger to the intake manifold (subscript $\mathrm{im}$ ) where the gas is delivered to the engine with a mass flow rate $Q_{e n g}$ and air fraction $F_{i m}$. In this section, we consider the problem of controlling the air fraction in the intake manifold by regulating the LP-EGR mass flow rate. We focus on the generation of the target $Q_{\text {egrl }}$, which then is translated into LP-EGR valve position by a secondary control. Since the EGR valves potision controllers have been already implemented in modern engines and is an application well known in the engine control community, then we do not detail its functionally in this work.

The admission air-path presented in Figure 1 can be considered as a series of tubes that are linked together by volumes, i.e. by the volumes upstream and downstream of the heat exchanger $V_{u h e}$ and $V_{d h e}$, respectively. Using this formulation, we can define three tube sections and three control volumes. The three tube sections are defined as: the tube downstream of the compressor (up to the heat exchanger), the heat exchanger with $N_{d}$ tubes in parallel and the tube downstream of the heat exchanger. The control volumes are: the tube upstream of the compressor (denoted as $V_{u c}$ where gas mixture occurs), the heat exchanger upstream volume $V_{u h e}$ and downstream volume $V_{d h e}$. The control volumes allow us to formulate a zero-dimensional model for the interfaces between the components of the air-path where most of the mass transport phenomenon occurs (the three tubes). In other words, we do not model the evolution of the states along the space dimensions in these volumes. Instead, we describe the dynamics of the flow using a volumeaverage formulation which eases the modeling of the air-path without affecting significantly the accuracy of the mass transport representation.

The one-dimensional modeling of the engine admission air-path is particularly complex as it involves solving the $1 \mathrm{D}$ Euler equations for a compressible gas [36], which is not appropriate for designing real-time feedback strategies. Therefore, the following assumptions are made to simplify the complexity of the air fraction model, making it suitable for Diesel engine control.

Assumption 2: We assume the following:

A-21: the pressure and particle speed dynamics are much faster than the air fraction dynamics; 
A-22: no friction and thermal losses are considered (except for the heat exchanger);

A-23: the speed density equation (equation that accurately predicts the amount of air ingested by an engine during the induction stroke [34]) is considered to model the engine intake mass flow rate. No pulsating flow is considered;

A-24: the cross-sectional area of each of the air-path sections (tube between two devices) is considered constant along the space variable $x$.

The air fraction in a control volume is modeled by considering the classical OD approach [35]:

$$
\dot{F}_{v}=\frac{R T_{v}}{p_{v} V_{v}}\left(\sum_{i=1}^{n_{i}}\left(Q_{i} F_{i}\right)-\sum_{o=1}^{n_{o}}\left(Q_{o} F_{v}\right)\right)
$$

where $F_{v}, T_{v}, p_{v}, V_{v}$ are the control volume air fraction, temperature, pressure and volume, respectively. $n_{i}$ and $n_{o}$ are the amount of mass inputs and outputs and $Q_{i}$ and $Q_{o}$ are the input and output mass flow rates, respectively. Assumption A-21 implies that $Q_{e n g}=Q_{\text {air }}+Q_{\text {egrl }}$. Therefore, the following equations describe the air mass fraction dynamics in the tube upstream of the compressor and in both heat exchanger volumes (according to (26)):

$$
\begin{gathered}
\dot{F}_{u c}=\frac{R T_{u c}}{p_{u c} V_{u c}}\left(-\left(Q_{\text {egrl }}+Q_{a i r}\right) F_{u c}+Q_{\text {egrl }} F_{\text {egrl }}+Q_{\text {air }}\right) \\
\dot{F}_{u h e}=\frac{R T_{d c}}{p_{d c} V_{u h e}}\left(-\left(Q_{\text {egrl }}+Q_{a i r}\right) F_{u h e}+\left(Q_{\text {egrl }}+Q_{a i r}\right) F_{d c}\left(L_{d c}, t\right)\right) \\
\dot{F}_{\text {dhe }}=\frac{R T_{d h e}}{p_{d h e} V_{d h e}}\left(-\left(Q_{e g r l}+Q_{a i r}\right) F_{d h e}+\left(Q_{\text {egrl }}+Q_{a i r}\right) F_{h e}\left(L_{h e}, t\right)\right)
\end{gathered}
$$

where $F_{u c}, T_{u c}$ and $p_{u c}$ are the air fraction, temperature, pressure and volume upstream of the compressor. $F_{\text {uhe }}$ and $F_{\text {dhe }}$ are the air fractions upstream and downstream of the heat exchanger, respectively. $F_{h e}$ is the air fraction inside the heat exchanger and $F_{d c}\left(L_{d c}, t\right)$ and $F_{h e}\left(L_{h e}, t\right)$ are the output air fractions of the compressor downstream tube and the heat exchanger tubes, respectively. Note that the dynamics of (27) - (29) are defined in terms of the states with the exception of (27) that depends on the time-varying additive term $Q_{\text {egrl }} F_{\text {egrl }}+Q_{a i r}$. This issue is addressed in the sequel to obtain a system in the form of the dynamic boundary conditions (3). 
For the 1D model of the air fraction dynamics in the tube sections, consider the change in mass fraction across a control volume of length $\partial x$, which can be expressed as [36]:

$$
\partial_{t}\left[\rho(x, t) A_{t}(x) F(x, t)\right]+\partial_{x}\left[\rho(x, t) u(x, t) A_{t}(x) F(x, t)\right]=0, \quad \forall x \in[0, L], t \geq 0
$$

where $L$ is the length of the pipe, $A_{t}$ the tube cross section, $\rho$ the gas density and $u$ the speed of particles. Equation (30) can be expressed as (e.g. see [36] Chapter 4.2.5 for more details):

$$
\partial_{t}[\rho(x, t) F(x, t)]+\partial_{x}[\rho(x, t) u(x, t) F(x, t)]+\rho(x, t) u(x, t) F(x, t) \partial_{x}\left[\ln \left(A_{t}(x)\right)\right]=0
$$

Expanding (31) and dividing by $F(x, t)$ gives:

$$
\begin{aligned}
& \partial_{t} \rho(x, t)+u(x, t) \partial_{x} \rho(x, t)+\rho(x, t) \partial_{x} u(x, t)+\rho(x, t) u(x, t) \partial_{x}\left(\ln \left(A_{t}(x)\right)\right) \\
& +\frac{\rho(x, t)}{F(x, t)} \partial_{t} F(x, t)+\frac{\rho(x, t) u(x, t)}{F(x, t)} \partial_{x} F(x, t)=0
\end{aligned}
$$

The first four terms of (32) constitute the global continuity of mass implying, that the sum of the four terms equals zero. Therefore, the air fraction continuity equation implies that:

$$
\begin{gathered}
\partial_{t} F(x, t)+u(x, t) \partial_{x} F(x, t)=0 \\
F(0, t)=F_{\text {in }}(t) \quad F(x, 0)=F_{0}(x), \quad \forall x \in[0, L], t \geq 0
\end{gathered}
$$

where $F_{\text {in }}$ is the air fraction at the input boundary condition, $F_{0}(x)$ is a continuous differentiable function describing the initial conditions and $u(t, x)$ is the air fraction propagation speed (independent of the air fraction).

The assumptions A-21, A-22 and A-24 imply that $u(x, t)$ is only time-varying (constant in space), which simplifies significantly the solution of (33). For the heat exchanger, where A-22 does not apply, the average temperature and pressure are defined as follows:

$$
T_{h e}=\frac{T_{d c}+T_{d h e}}{2}, \quad p_{h e}=\frac{p_{d c}+p_{d h e}}{2}
$$

where $T_{h e}$ and $p_{h e}$ are the heat exchanger temperature and pressure, respectively. Uniform pressure and temperature distribution are assumed inside the heat exchanger to obtain (34), which 
allows approximating a space average particle speed inside the heat exchanger as presented later in (38).

From (33) and (34), the dynamics of each tube section can be modeled with the following set of time-varying first order hyperbolic partial differential equations:

$$
\begin{gathered}
\partial_{t} F_{d c}+u_{d c}(t) \partial_{x} F_{d c}=0, \quad F_{d c}(0, t)=F_{u c}(t), \quad F_{d c}(x, 0)=F_{d c 0}(x) \\
\partial_{t} F_{h e}+u_{h e}(t) \partial_{x} F_{h e}=0, \quad F_{h e}(0, t)=F_{u h e}(t), \quad F_{h e}(x, 0)=F_{h e 0}(x) \\
\partial_{t} F_{d h e}+u_{d h e}(t) \partial_{x} F_{d h e}=0, \quad F_{d h e}(0, t)=F_{d h e}(t), \quad F_{d h e}(0, x)=F_{d e 0}(x)
\end{gathered}
$$

for all $x \in[0,1]$ and $t \geq 0$. The particle speeds for each air-path section $u_{d c}, u_{h e}$ and $u_{d h e}$ can be normalized (implying that $x \in[0,1]$ ) and calculated using the ideal gas law as follows:

$$
u_{d c}=\frac{R T_{d c}\left(Q_{a i r}+Q_{e g r l}\right)}{p_{d c} A_{d c} L_{d c}}, \quad u_{h e}=\frac{R T_{h e}\left(Q_{a i r}+Q_{e g r l}\right)}{p_{h e} A_{h e} L_{h e} N_{d}}, \quad u_{d h e}=\frac{R T_{d h e}\left(Q_{a i r}+Q_{e g r l}\right)}{p_{d h e} A_{d h e} L_{d h e}}
$$

where $L_{d c}, L_{h e}$ and $L_{d h e}$ are the lengths, $A_{d c}, A_{h e}$ and $A_{d h e}$ are the cross-sectional areas of each of the respective tube sections and $N_{d}$ is the number of parallel tubes in the heat exchanger. To use the results of Theorem 1, the system has to be in the form of (1) with boundary conditions (3). System (35) - (37) is a cascade of hyperbolic systems connected by the dynamics defined by (27) - (29), which does not correspond to the system structure (1). This problem is solved by following a similar approach as in [18], where $n$ hyperbolic systems in cascade form are combined in one PDE of order $n$ by re-defining the boundary control. However, it is important to highlight that our realization takes into account the dynamic behavior of the boundary conditions, therefore a different stability analysis as to be taken into account. Consider the definition of the air fraction errors as:

$$
\begin{gathered}
\xi_{d c}(x, t)=F_{d c}(x, t)-F_{\text {imref }}, \quad \xi_{h e}(x, t)=F_{h e}(x, t)-F_{\text {imref }}, \\
\xi_{d h e}(x, t)=F_{d c}(x, t)-F_{\text {imref }},
\end{gathered}
$$


and

$$
\xi_{\text {uc }}(t)=F_{\text {uc }}(t)-F_{\text {imref }}, \quad \xi_{\text {uhe }}(t)=F_{\text {uhe }}(t)-F_{\text {imref }}, \quad \xi_{\text {dhe }}(t)=F_{\text {dhe }}(t)-F_{\text {imref }}
$$

where $F_{\text {imref }}$ is a given scalar reference for the air fraction. According to (39) and (40), we can rewrite the dynamics of the simplified air fraction air-path model (27) - (29) and (35) - (37) as an LPV hyperbolic PDE of order $n$ as follows:

$$
\partial_{t}\left[\begin{array}{c}
\xi_{d c} \\
\xi_{h e} \\
\xi_{d h e}
\end{array}\right]+\left[\begin{array}{ccc}
\frac{\varphi_{2}}{A_{d c} L_{d c}} & 0 & 0 \\
0 & \frac{\varphi_{3}}{A_{h e} L_{h e} N_{d}} & 0 \\
0 & 0 & \frac{\varphi_{4}}{A_{\text {dhe }} L_{\text {dhe }}}
\end{array}\right] \partial_{x}\left[\begin{array}{c}
\xi_{d c} \\
\xi_{h e} \\
\xi_{\text {dhe }}
\end{array}\right]=0
$$

with the dynamic boundary conditions in the form of (3):

$$
\left[\begin{array}{c}
\dot{\xi}_{u c} \\
\dot{\xi}_{u h e} \\
\dot{\xi}_{d h e}
\end{array}\right]=\left[\begin{array}{ccc}
-\frac{\varphi_{1}}{V_{u c}} & 0 & 0 \\
0 & -\frac{\varphi_{2}}{V_{u h e}} & 0 \\
0 & 0 & -\frac{\varphi_{4}}{V_{d h e}}
\end{array}\right]\left[\begin{array}{c}
\xi_{u c} \\
\xi_{u h e} \\
\xi_{d h e}
\end{array}\right]+\left[\begin{array}{ccc}
\frac{\varphi_{1}}{V_{u c}} & 0 & 0 \\
0 & \frac{\varphi_{2}}{V_{u h e}} & 0 \\
0 & 0 & \frac{\varphi_{4}}{V_{d h e}}
\end{array}\right]\left[\begin{array}{c}
\tilde{v} \\
\xi_{d c}(1) \\
\xi_{h e}(1)
\end{array}\right]
$$

The boundary control in the form of (4) that allows writing the cascade of hyperbolic systems as a PDE of order $n$ is:

$$
\left[\begin{array}{c}
\tilde{v} \\
\xi_{d c}(1) \\
\xi_{h e}(1)
\end{array}\right]=\left[\begin{array}{ccc}
0 & 0 & K_{13} \\
1 & 0 & 0 \\
0 & 1 & 0
\end{array}\right]\left[\begin{array}{c}
\xi_{d c}(1) \\
\xi_{h e}(1) \\
\xi_{d h e}(1)
\end{array}\right]
$$

where $\tilde{v}$ is a virtual control input that cancels the additive terms of (27) and is defined as:

$$
\tilde{v}=\frac{F_{\text {egrl }} Q_{\text {egrl }}}{Q_{\text {air }}+Q_{\text {egrl }}}-F_{\text {imref }}-\frac{Q_{a i r}}{Q_{a i r}+Q_{\text {egrl }}}
$$

The time-varying parameters $\varphi_{i}$ are given by: 


$$
\begin{aligned}
& \varphi_{1}=\frac{R T_{u c}\left(Q_{a i r}+Q_{\text {egrl }}\right)}{p_{u c}}, \quad \varphi_{2}=\frac{R T_{d c}\left(Q_{a i r}+Q_{\text {egrl }}\right)}{p_{d c}}, \\
& \varphi_{3}=\frac{R T_{h e}\left(Q_{a i r}+Q_{\text {egrl }}\right)}{p_{h e}}, \quad \varphi_{4}=\frac{R T_{\text {dhe }}\left(Q_{\text {air }}+Q_{\text {egrl }}\right)}{p_{\text {dhe }}}
\end{aligned}
$$

From (43) and (44), the LP-EGR mass flow rate is defined as:

$$
Q_{\text {egrl }}=\frac{Q_{a i r}\left(F_{\text {imref }}-1+K_{13}\left(\xi_{\text {dhe }}(1)\right)\right)}{F_{\text {egrl }}-F_{\text {imref }}-K_{13}\left(\xi_{\text {dhe }}(1)\right)}
$$

The LP-EGR mass flow rate target of (46) is then transformed into LP-EGR valve position by a secondary controller which typically is composed by an open-loop control obtained from an inverted orifice equation and a closed-loop control composed by a PID and a LP-EGR mass flow rate estimation as the one presented in [13]. Figure 2 presents the final schematic of the proposed air fraction control in the intake manifold.

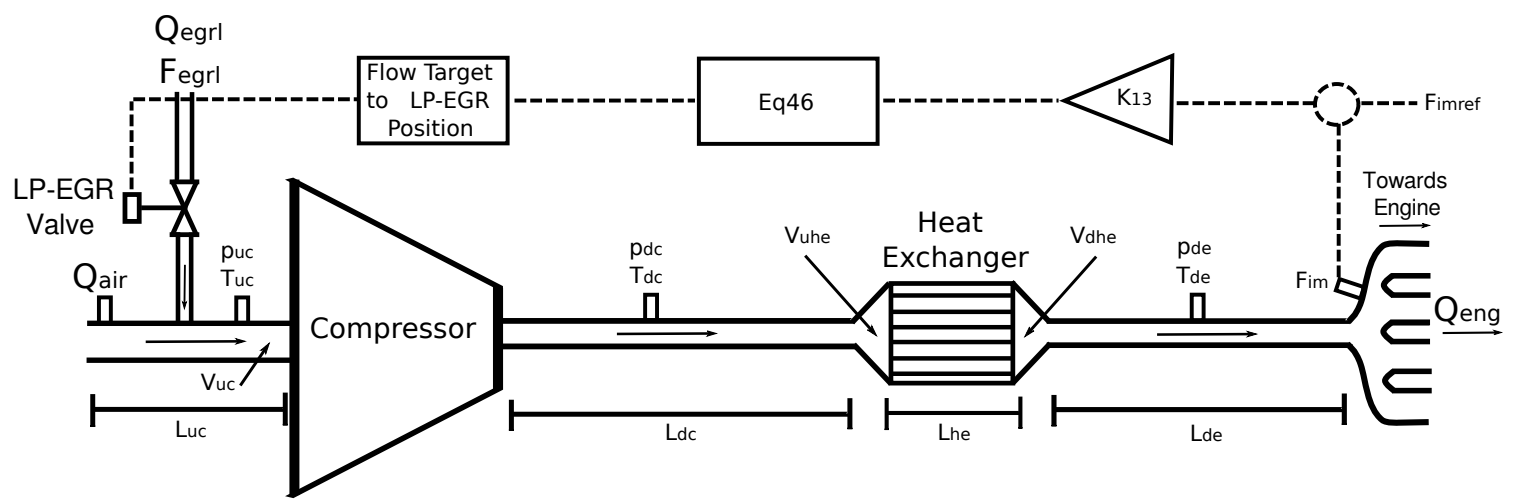

Fig. 2: Resulting air fraction control architecture

The system (41) - (45) with boundary control (43) is in the adequate form to apply the results of Theorem 1. However, with the definition of the varying parameters presented in (45), the polytope (convex set $Z_{\varphi}$ ) obtained by using the polytopic linear representations (PLR) (12) and (13) is very large and therefore highly conservative. Since all the varying parameters in (45) are strongly dependent on the compressor mass flow rate $Q_{\text {air }}+Q_{\text {egrl }}$, specially from the fact that this flow has a direct impact on the mass transport time in the admission path, it is highly convenient to use this quantity to reduce the size of the polytope and therefore the conservatism 
of the results. The engine speed could be also used to represent the dynamics of the admission path [14]; however, it is more complicated in this application because other factors have to be taken into account to express the mass transport phenomenon in terms of $N_{\text {eng }}$. Among these factors we find the high-pressure EGR, the swirl and the engine volumetric efficiency.

Let us redefine the varying parameters as:

$$
\tilde{\varphi}(t)=\left[Q_{\text {air }}+Q_{\text {egrl }},\left(Q_{\text {air }}+Q_{\text {egrl }}\right)^{2}\right]
$$

and the original varying parameters $\varphi$ as a linear combination of $\tilde{\varphi}$ as follows:

$$
\varphi_{j}(t)=\vartheta_{j}^{T} \psi(t), \quad j \in[1, \ldots, 4]
$$

where $\vartheta_{j} \in \mathbb{R}^{3}$ is an unknown coefficient vector and $\psi(t)=\left[1,\left(Q_{\text {air }}+Q_{\text {egrl }}\right),\left(Q_{\text {air }}+Q_{\text {egrl }}\right)^{2}\right]$. A classical least square method can be used to determine the optimal set of coefficients $\vartheta_{j}$ such that $\left|\varphi_{j}(t)-\vartheta_{j}^{T} \psi(t)\right|^{2}$ is minimized and the coefficient identification can be done using engine benchmark measurements over representative engine operating conditions. With this approach, the amount of varying parameters is decreased from four to two, which significantly reduces the conservatism of the control synthesis.

In Figure 3a, the polytope $Z_{\varphi}$ formed by all the extremities of $\tilde{\varphi}_{1}$ and $\tilde{\varphi}_{2}$ is shown. As proposed demonstrated in [29], the conservatism can be further reduced from the fact that $\tilde{\varphi}_{2}=\tilde{\varphi}_{1}^{2}$, which allows considering only the polytope formed by three vertexes for the control synthesis, as shown in Figure 3b.

The bounds of the varying parameter vector $\tilde{\varphi}$ can also be found using experimental measurements over representative engine operating conditions.

To summarize, the air fraction is controlled using the results of Section II over the polytope formed by the extremities of $\tilde{\varphi}$ to find the gain $K_{13}$ in (46) such that the air fraction at the engine intake manifold $F_{i m}$ converges in finite time to the reference $F_{\text {imref }}$. In the next section, simulation results illustrate the performance of the proposed air fraction control. 


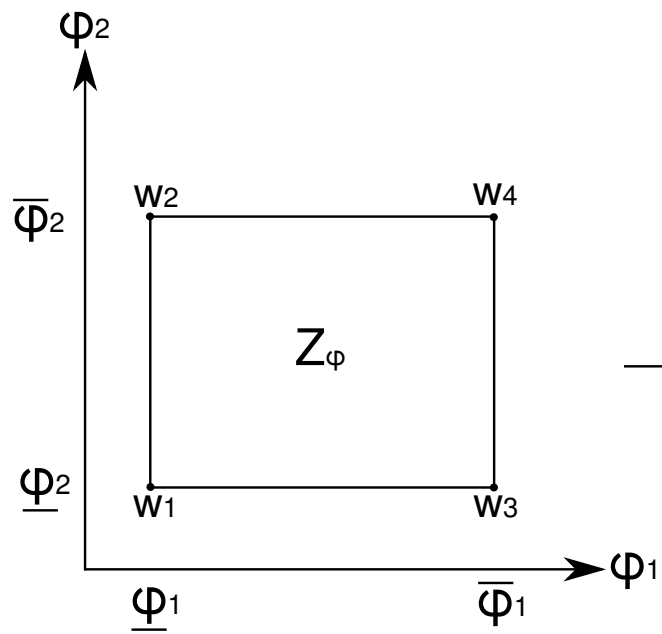

(a) Original parameter set

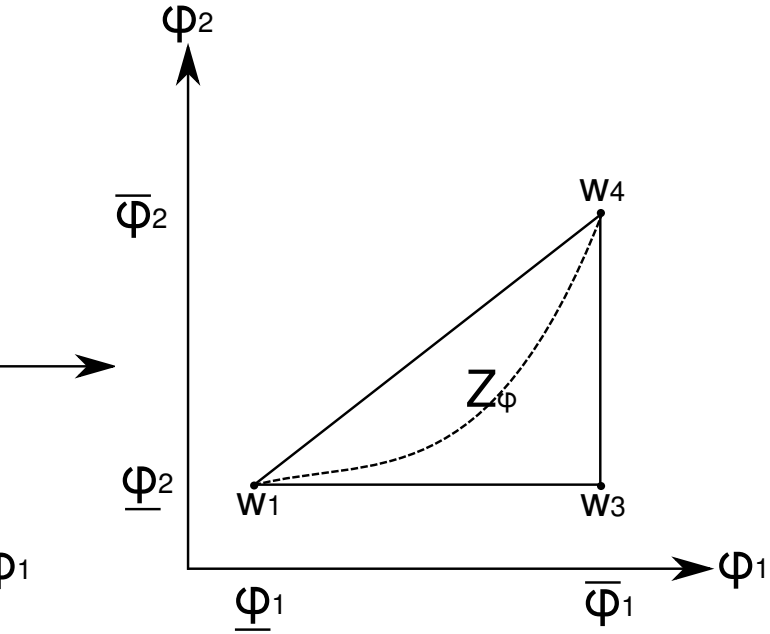

(b) Reduced parameter set

Fig. 3: Parameter set reduction

\section{Simulation Results}

To illustrate the effectiveness of the proposed LPV boundary control strategy, an air fraction control (46) that satisfies Theorem 1 is simulated along with the setup shown in Figure 1. The simulation is performed using a 1D model based on the Euler equations for compressible gases, which is numerically solved with a MacCormack numerical scheme and a Time Variation Diminishing strategy (for more detail on the numerical methods refer to $[36,15,33]$ ). This $1 \mathrm{D}$ model has been experimentally validated for inflow, outflow and intra-pipe restrictions [9] [12] [25]. Table I gives the physical and numerical parameters used for the simulation of a light-duty 1.6 liter Diesel engine.

The bounds on each parameter as well as the coefficients of (48) are found from measurements on an engine benchmark operated over a wide range of engine operating conditions. More precisely, we use the new motor vehicle emissions group cycle along with two additional engine cycles. The bounds obtained for $\tilde{\varphi}$ are the following:

$$
\tilde{\varphi_{1}} \in[0.009,0.074], \quad \tilde{\varphi_{2}} \in[0.00009,0.0054]
$$




\begin{tabular}{|c|c|c|c|}
\hline Variable & Value & Variable & Value \\
\hline$L_{d c}$ & $1.3 \mathrm{~m}$ & $A_{\text {he }}$ & $6 \times 10^{-4} \mathrm{~m}^{2}$ \\
$A_{d c}$ & $0.002 \mathrm{~m}^{2}$ & $V_{\text {uhe }}$ & $5 \times 10^{-4} \mathrm{~m}^{3}$ \\
$V_{u c}$ & $3 \times 10^{-4} \mathrm{~m}^{3}$ & $V_{d h e}$ & $5 \times 10^{-4} \mathrm{~m}^{3}$ \\
$N_{d}$ & 8 & $L_{d h e}$ & $1.1 \mathrm{~m}$ \\
$L_{h e}$ & $0.58 \mathrm{~m}$ & $A_{d h e}$ & $0.002 \mathrm{~m}^{2}$ \\
$d t$ & $40 \mu \mathrm{s}$ & $N_{\text {uhe }}$ & 50 \\
$N_{h e}$ & 20 & $N_{\text {dhe }}$ & 50 \\
\hline
\end{tabular}

TABLE I: Simulation parameters. $d t$ is the simulation time step and $N_{u h e}, N_{h e}$ and $N_{d h e}$ are the discretization sizes of the tube upstream the compressor, heat exchanger and downstream the compressor, respectively

The identification of the coefficient vector $\vartheta_{i}$ is performed using a traditional least-squaredmethod and the Penrose-Moore pseudo-inverse. Table II presents the identified root-mean-square deviation (RMSD) of $\vartheta_{i}$, where $R M S D_{j}=R M S D\left(\varphi_{j}(t)-\vartheta_{j}^{T} \psi(t)\right)$.

\begin{tabular}{|c|c|}
\hline Variable & Value \\
\hline$R M S D_{1}$ & $0.1 \%$ \\
$R M S D_{2}$ & $4.1 \%$ \\
$R M S D_{3}$ & $2.9 \%$ \\
$R M S D_{4}$ & $4.7 \%$ \\
\hline
\end{tabular}

TABLE II: The RMSD obtained after the solution of the least square method

As shown in Table II, the parametrization (48) is representative of the engine varying parameters $\varphi$ and is therefore adequate for the boundary control synthesis.

Consider, as a criterion for the boundary control design, a maximum air fraction convergence time of $1.2 \mathrm{~s}$, (i.e. the time to reach $90 \%$ of the final asymptotic value). From (21) and the smallest eigenvalue of $\Lambda(\varphi)$, we find that $\mu>0.6$ is required to achieve the desired convergence time. Solving (14) over the reduced polytope (presented in Figure 3b), leads to the following control gain and Lyapunov matrices $P_{1}$ and $P_{2}$ : 


$$
K_{13}=0.25, \quad P_{1}=\left[\begin{array}{ccc}
0.0422 & 0 & 0 \\
0 & 0.0493 & 0 \\
0 & 0 & 0.0353
\end{array}\right], \quad P_{2}=\left[\begin{array}{ccc}
0.445 & 0 & 0 \\
0 & 0.299 & 0 \\
0 & 0 & 0.129
\end{array}\right]
$$

Figures 4 and 5 show the simulation results obtained from a change of air fraction reference ( $F_{\text {imref }}$ ) from 1 to 0.7 at time $0.1 \mathrm{~s}$. Three different air-path operating conditions are simulated, in order to verify the robustness of the controller with respect to parameter variation and the impact of the mass transport phenomenon in the control of the air fraction in the intake manifold.

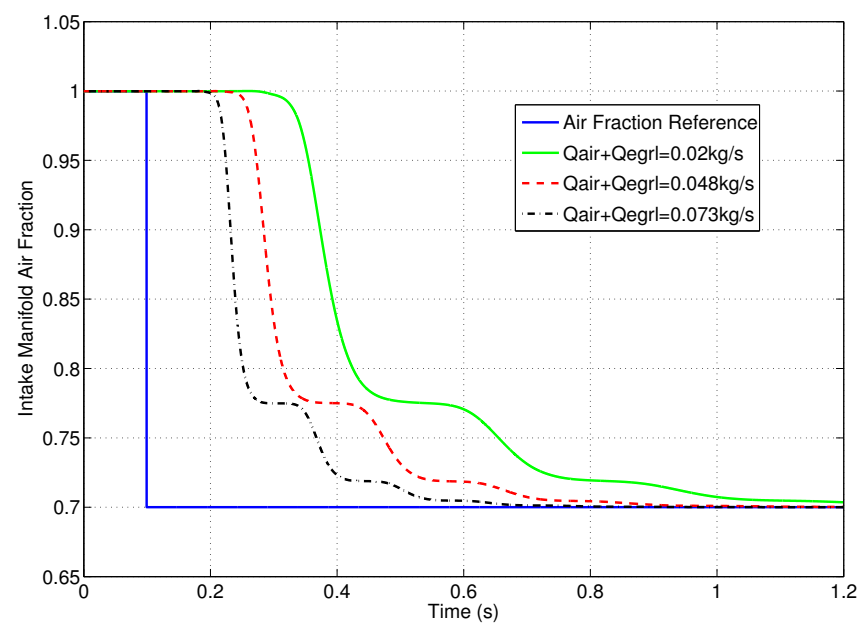

Fig. 4: Intake manifold air fraction simulation results for a change of air fraction reference

As depicted in Figure 4, the intake manifold air fraction converges to the reference while respecting the convergence time criterion for the three operating conditions. The convergence time depends on the mass flow rate going through the air system (see Figure 4), which is due to the intrinsic time delays associated with transport in the system. In Figure 5 the LP-EGR mass flow rate is presented for the three engine operating conditions: when the mass flow rate increases in the system, $Q_{\text {egrl }}$ also does, to maintain the appropriate air fraction.

The convergence of the Lyapunov function (16) is illustrated on Figure 6, where it is shown that the actual system convergence is always faster than the exponential decrease predicted by (21). 


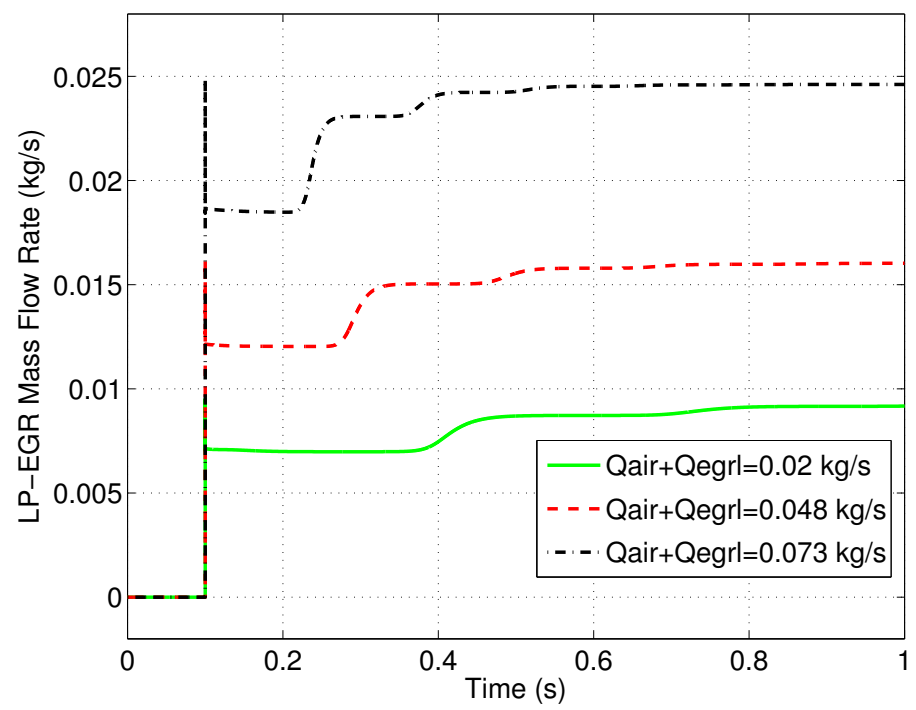

Fig. 5: LP-EGR mass flow rate simulation results for a change of air fraction reference

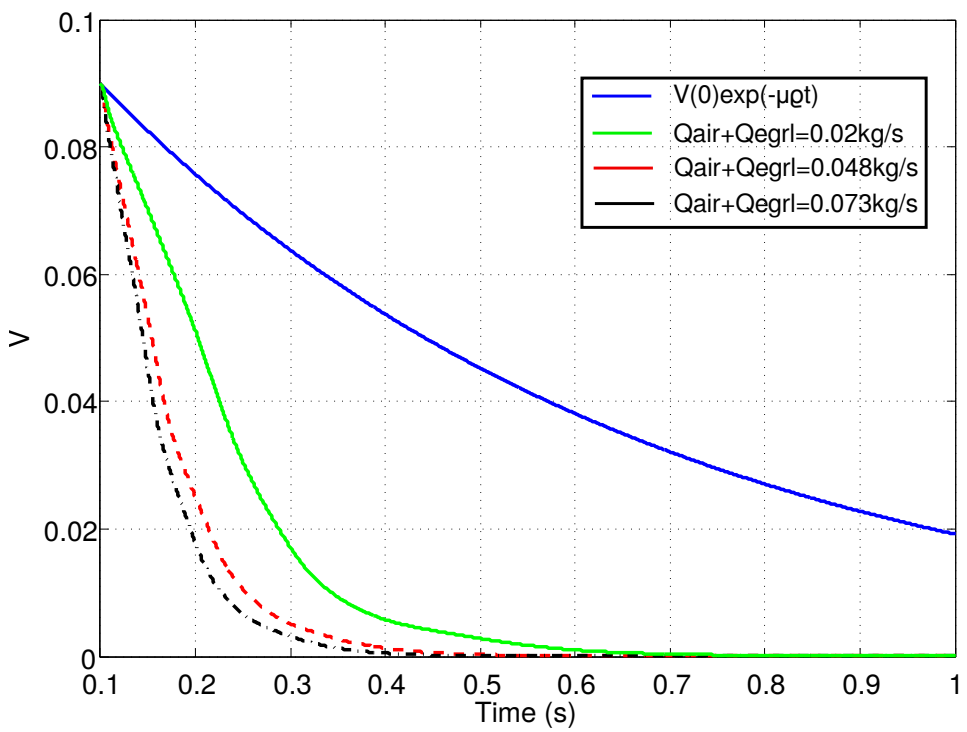

Fig. 6: Convergence of the Lyapunov function

To evaluate the efficiency of the controller under strong engine transient conditions, a simulation of an engine cycle with abrupt changes in fuel quantities and air fraction in the exhaust and intake manifold has been performed. Figure 7 shows the engine operating conditions during the proposed maneuver. 

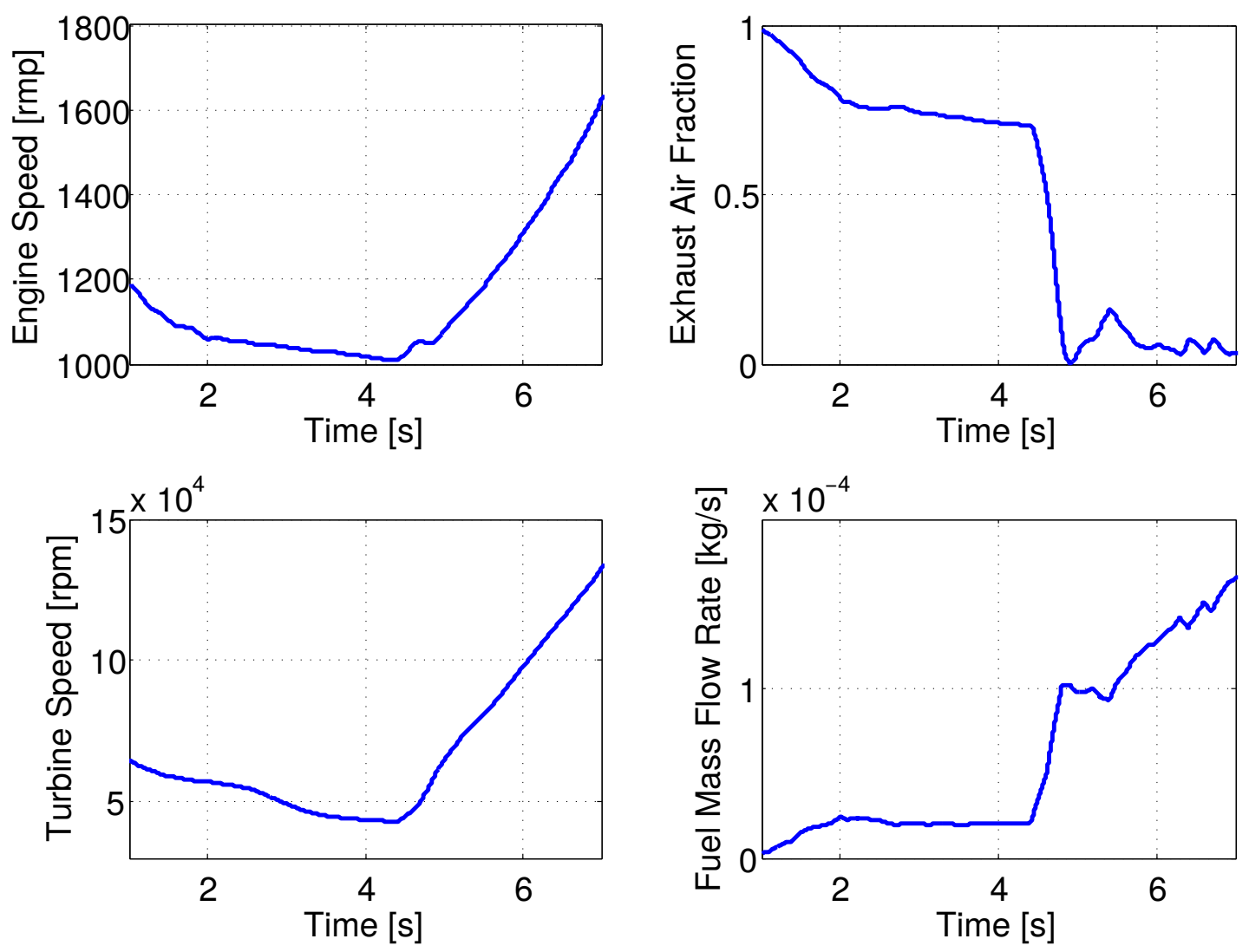

Fig. 7: Time evolution of the engine operating conditions

Figures 8 and 9 present the evolution in time of the air fraction in the intake manifold and the LP-EGR mass flow rate, respectively. As depicted, the air fraction in the intake manifold effectively tracks the air fraction target $F_{\text {imref }}$ in a smooth trajectory, only separated by the transport time inherent of the system. Figure 9 shows that also the $Q_{\text {egrl }}$ effectively compensates the variation of the engine operating conditions, enabling the controller to properly control the air fraction in the intake manifold.

Due to the low calculation load and low complexity of the boundary control (46), this approach is suitable for real-time implementation in a production engine. The current limitation to implement this strategy mainly comes from the lack of reliable measurements or estimations of the fresh air mass fraction in the intake manifold under strong transient conditions (e.g. lack of a reliable sensor for detecting the mass transport). However, many efforts are being done to address this 


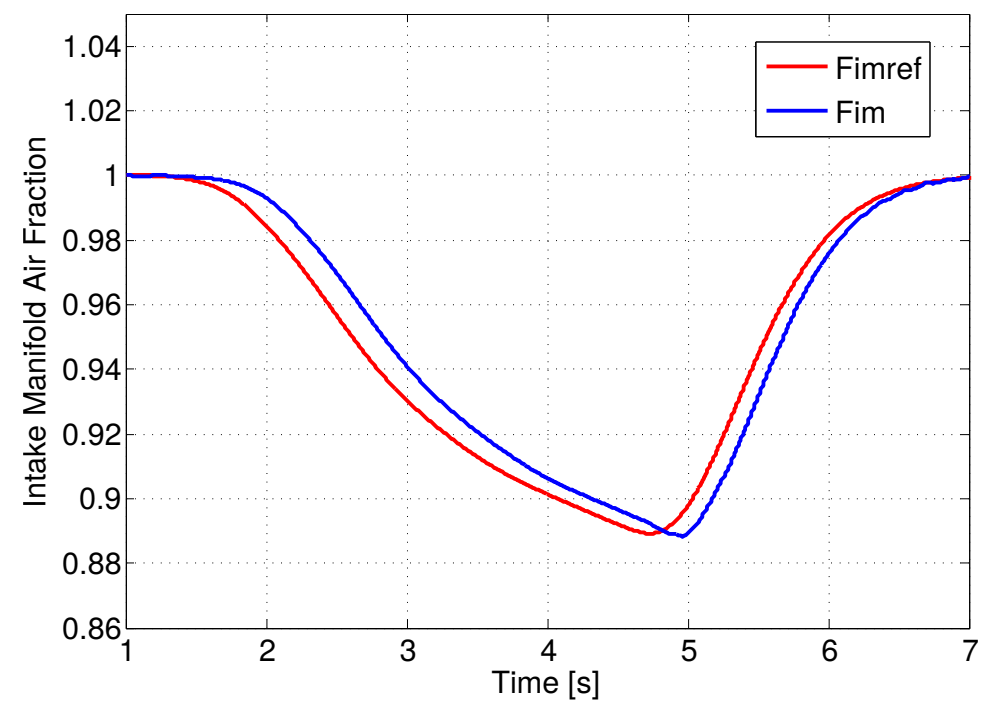

Fig. 8: Time evolution of the air fraction in the intake manifold

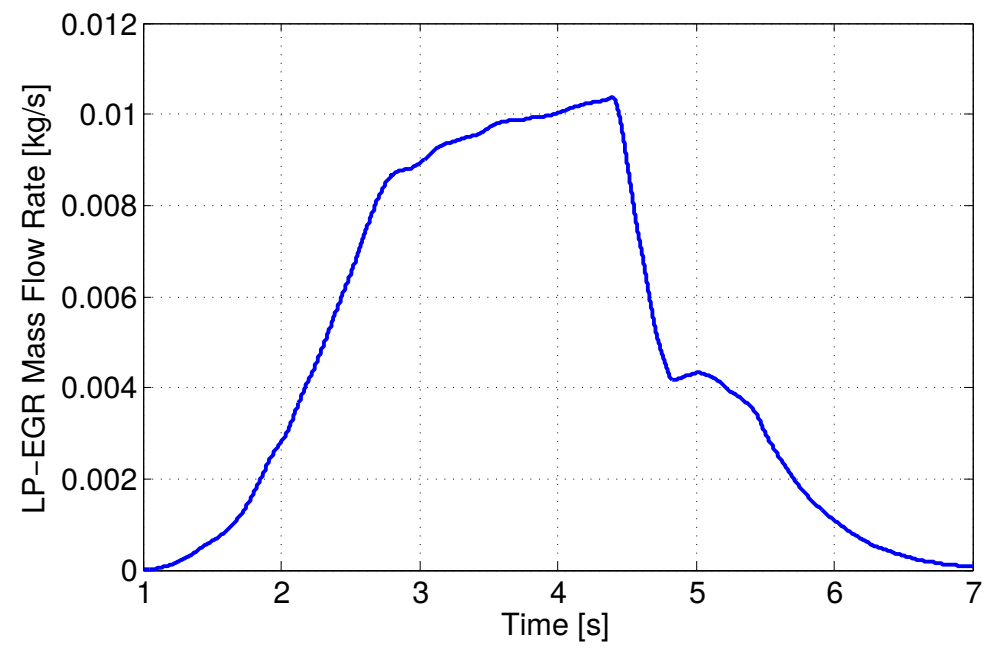

Fig. 9: Time evolution of the LP-EGR mas flow rate

issue by using air fraction observers that take into account the mass transport phenomenon (e.g. [11]), which would enable the implementation of the proposed boundary control in production engines. 


\section{Conclusions And Perspectives}

This paper proposes a new solution for the boundary control of the fresh air mass fraction in a Diesel engine operated with low-pressure exhaust gas recirculation. A formulation using a cascade of first order LPV hyperbolic systems with dynamics associated with the boundary conditions has been considered to model the air fraction transport phenomenon. Some sufficient conditions for the exponential stability of such systems were obtained using a matrix inequality approach. The exponential stability was demonstrated by means of a strict Lyapunov function formulation, along with a linear polytopic representation for the LPV hyperbolic system.

By defining a minimal air fraction convergence time as the performance criterion, an air fraction regulation strategy was designed using the previous stability results. The conservatism of the boundary feedback control was significantly reduced by redefining the system varying parameters. The complexity of the obtained boundary control is very low, which makes it suitable for real-time implementation. A 1D simulation of the engine admission air-path with the proposed controller was performed to evaluate the effectiveness of the control approach. The simulation results are promising and motivate future steps toward implementation.

The new control design method presented in this paper has many applications in various systems governed by hyperbolic PDE's, such as mine ventilation, traffic control and hydraulic networks. However, many questions are still open. In particular, a generalization of some sufficient conditions for the exponential stability of hyperbolic systems with non-linear dynamic boundary conditions seems to be a challenging issue. Considering perturbations in system (1) and in the boundary conditions dynamics (3) is also a natural extension of this work.

\section{REFERENCES}

[1] K. Akihama, Y. Takatory, K. Inagaki, S. Sasaki, and A. Dean. Mechanism of the smokeless rich Diesel combustion by reducing temperature. SAE Technical Paper 2001-01-0655, doi:10.4271/2001-01-0655, 2001.

[2] M. Alriksson and I. Denbrantt. Low temperature combustion in a heavy duty Diesel engine using high levels of EGR. SAE Technical Paper 2006-01-0075, doi:10.4271/2006-01-0075, 2006. 
[3] G. Angelis. System Analysis, Modelling and Control with Polytopic Linear Models. PhD Thesis. Technische Universiteit Eindhoven, Eindhoven, 2001.

[4] G. Bastin, J-M. Coron, and B. d'Andréa Novel. Using hyperbolic systems of balance laws for modelling, control and stability analysis of physical networks. Lecture notes for the Pre-Congress Workshop on Complex Embedded and Networked Control Systems, 17th IFAC World Congress, Seoul, Korea, 2008.

[5] G. Bastin, J-M. Coron, and B. d'Andréa Novel. On Lyapunov stability of linearized SaintVenant equations for a sloping channel. Networks and Heterogeneous Media, 4(2):177-187, 2009.

[6] D. Bresch-Pietri, T. Leroy, J. Chauvin, and N. Petit. Prediction-based trajectory tracking of external gas recirculation for turbocharged SI engines. Proceedings of the American Control Conference, Montréal, Canada, pages 5718-5724, 2012.

[7] F. Castillo, E. Witrant, and L. Dugard. Contrôle de température dans un flux de Poiseuille. Proceedings of the IEEE Conférence Internationale Francophone d'Automatique, Grenoble, France, 2012.

[8] F. Castillo, E. Witrant, and L. Dugard. Dynamic boundary stabilization of linear parameter varying hyperbolic systems: Application to a Poiseuille flow. Proceedings of the 11th IFAC Workshop on Time-Delay Systems, Grenoble, France, 2013.

[9] F. Castillo, E. Witrant, L. Dugard, Vincent Talon, David Chalet, and Pascal Chesse. Intrapipe restriction non-homentropic boundary resolution method. SAE Technical Paper 201301-0582, 2013.

[10] F. Castillo, E. Witrant, C. Prieur, and L. Dugard. Dynamic boundary stabilization of linear and quasi-linear hyperbolic systems. Proceedings of the 51st IEEE Conference on Decision and Control, Maui, Hawai, pages 2952-2957, 2012.

[11] F. Castillo, E. Witrant, C. Prieur, and L. Dugard. Boundary observers for linear and quasilinear hyperbolic systems with application to flow control. Automatica, 49(11):3180-3188, 2013.

[12] F. Castillo, E. Witrant, V. Talon, and L. Dugard. Restriction model independent method for non-isentropic outflow valve boundary problem resolution. SAE Technical Paper 201201-0676, doi:10.4271/2012-01-0676, 2012.

[13] F. Castillo, E. Witrant, V. Talon, and L. Dugard. Simultaneous air fraction and low-pressure 
EGR mass flow rate estimation for Diesel engines. Proceedings of the 5th IFAC Symposium on System Structure and Control, Grenoble, France, 2013.

[14] J. Chauvin, G. Corde, N. Petit, and P. Rouchon. Motion planning for experimental airpath control of a Diesel homogeneous charge-compression ignition engine. Control Engineering Practice, 16:1081-1091, 2008.

[15] T.J. Chung. Computational Fluid Dynamics. Cambridge University Press, United Kingdom, 2002.

[16] J-M. Coron, G. Bastin, and B. d'Andréa Novel. Dissipative boundary conditions for onedimensional nonlinear hyperbolic systems. SIAM J. Control Optim, 47:1460-1498, 2008.

[17] J-M. Coron, B. d'Andréa Novel, and G. Bastin. A strict Lyapunov function for boundary control of hyperbolic systems of conservation laws. IEEE Transactions on Automatic control, 52:2-11, 2007.

[18] J. de Halleux. Boundary Control of Quasi-Linear Hyperbolic Initial Boundary-Value Problems. PhD Thesis. Presses universitaires de Louvain, Louvain la Neuve, Belgium, 2004.

[19] A. Diagne, G. Bastin, and J-M. Coron. Lyapunov exponential stability of 1-D linear hyperbolic systems of balance laws. Automatica, 48:109-114, 2012.

[20] O. Grondin, P. Moulin, and J. Chauvin. Control of a turbocharged Diesel engine fitted with high pressure and low pressure exhaust gas recirculation systems. 48th IEEE Conference on Decision and Control, Shanghai, China, pages 6582-6589, 2009.

[21] F.M. Hante and M. Sigalotti. Converse Lyapunov theorems for switched systems in Banach and Hilbert spaces. SIAM Journal on Control and Optimization, 49(2):752-770, 2011.

[22] A. Hribernik. The potential of the high and low-pressure exhaust gas recirculation. Proceedings of the SAE conference, Paper 2002-04-0029, 2002.

[23] M. Krstic and A. Smyshlyaev. Backstepping boundary control for first-order hyperbolic PDEs and application to systems with actuator and sensor delays. Systems and Control Letters, 57(9):750-758, 2008.

[24] T.T. Li. Controllability and observability for quasilinear hyperbolic systems. High Education Press, Beijing, 3, 2012.

[25] G. Martin. O-D -1-D Modeling of the air path of ICE Engines for control purposes. PhD thesis, Université d'Orléans, France, 2010. 
[26] F. Di Meglio, R. Vazquez, M. Krstic, and N. Petit. Backstepping stabilization of an underactuated $3 \times 3$ linear hyperbolic system of fluid flow transport equations. Proceedings of the American Control Conference, Montréal, Canada, pages 3365-3370, 2012.

[27] C. Prieur, A. Girard, and E. Witrant. Stability of switched linear hyperbolic systems by Lyapunov techniques. IEEE Transactions on Automatic control, to appear, 2014.

[28] C. Prieur and F. Mazenc. ISS-Lyapunov functions for time-varying hyperbolic systems of balance laws. Mathematics of Control, Signals and Systems, 24(1):111-134, 2012.

[29] D. Robert, O. Sename, and D. Simon. An H infinity LPV design for sampling varying controllers: Experimentation with a T-inverted pendulum. IEEE Transaction on Control, Systems Technology, 18:741-750, 2010.

[30] T. Ryan and A. Matheaus. Fuel requirements for HCCI engine operation. SAE transactionsJournal of Fuels Lubricants, 112:1143-1152, 2003.

[31] V. Dos Santos and C. Prieur. Boundary control of open channels with numerical and experimental validations. IEEE Transactions on Control Systems Technology, 16(6):12521264, 2008.

[32] V. Dos Santos, M. Rodrigues, and M. Diagne. A multi-model approach to Saint-Venant equations: A stability study by LMIs. Int. J. Appl. Math. Comput. Sci, 22(3):539-550, 2012.

[33] J. Tannehill, D. Anderson, and R. Pletcher. Computational Fluid Mechanics and Heat Transfer. Taylor and Francis, Washington DC, USA, 1997.

[34] J. Wang. Hybrid robust control for engines running low temperature combustion and conventional Diesel combustion modes. IEEE Transactions on Control Systems Technology, 16(6):1138-1151, 2007.

[35] J. Wang. Air fraction estimation for multiple combustion mode Diesel engines with dualloop EGR systems. Control Engineering and Practice 16, 1479-1468, 16, 2008.

[36] D.E. Winterbone and R.J. Pearson. Theory of Engine Manifold Design: Wave Action Methods for IC Engines. Society of Automotive Engineers. Inc, 2000.

[37] F. Yan and J. Wang. Control of a dual loop EGR air-path systems for advanced combustion Diesel engines by singular perturbation methodology. Proceedings of the American Control Conference, San Francisco, USA, pages 1561-1566, 2011. 